\title{
A Terapia Ocupacional e a dor crônica em pacientes de Ortopedia e Reumatologia: revisáo bibliográfica ${ }^{1}$
}

\author{
Marilles Fuchs ${ }^{\mathrm{a}}$, Marina Redekop Cassapian ${ }^{\mathrm{b}}$ \\ aTerapeuta Ocupacional, Graduada, Universidade Federal do Paraná - UFPR, Curitiba, PR, Brasil \\ ${ }^{\mathrm{b}}$ Terapeuta Ocupacional, Docente, Curso de Terapia Ocupacional, \\ Universidade Federal do Paraná - UFPR, Curitiba, PR, Brasil
}

\begin{abstract}
Resumo: A dor é considerada uma experiência individual desagradável e multidimensional. Quando crônica, impacta no desempenho ocupacional e, consequentemente, na qualidade de vida de quem a sente. Em razão da sua multidimensionalidade, faz-se necessária a intervenção multidisciplinar, na qual se insere o Terapeuta Ocupacional. O objetivo desta pesquisa foi averiguar os tipos de procedimentos adotados pelos Terapeutas Ocupacionais brasileiros e do exterior para intervir junto a pacientes com lesões ortopédicas e doenças reumáticas, que apresentam dor crônica. Para a elaboração deste trabalho, realizou-se revisão de literatura, com pesquisa de referenciais teóricos no acervo da Biblioteca de Ciências da Saúde da UFPR e nas bases de dados Lilacs, SciELO, Medline, OTseeker e OTdbase, publicados no período de 1999 a 2009. Os artigos selecionados para a discussão foram desenvolvidos por Terapeutas Ocupacionais que atuam nas áreas de Ortopedia e Reumatologia com pacientes acometidos por processos dolorosos crônicos. Com a realização da pesquisa, foi possível constatar que o Terapeuta Ocupacional é um importante componente da equipe multidisciplinar, com papel fundamental, uma vez que o foco de sua intervenção engloba o máximo desempenho ocupacional - com autonomia e independência - nas atividades cotidianas.
\end{abstract}

Palavras-chave: Dor, Terapia Ocupacional, Reumatologia, Ortopedia.

\section{Occupational Therapy and chronic pain with Orthopedics and Rheumatology patients: a literature review}

\begin{abstract}
Pain is considered an unpleasant individual multidimensional experience and it impairs occupational performance when chronic; consequently, it lowers the quality of life of individuals. Concerning its multidimensionality, a multidisciplinary approach, where the occupational therapist is inserted, is needed. The purpose of this study was to exploit the types of procedures used by occupational therapists in Brazil and abroad to intervene with patients with orthopedic injuries and rheumatic diseases with chronic pain. A literature revision was performed for the elaboration of this study, researching theoretical references at "Ciências da Saúde" collection at the Federal University of Paraiba library and in the following databases: Lilacs, SciELO, Medline, OTseeker and OTdbase, published in the time period between 1999 and 2009. The articles selected for discussion were developed by occupational therapists working in the fields of orthopedics and rheumatology with patients suffering from chronic painful processes. It was possible to verify that the occupational therapist is an important component of the multidisciplinary team, playing a fundamental role, once the focus of its intervention includes the maximum occupational acting - with autonomy and independence - in the daily activities.
\end{abstract}

Keywords: Pain, Occupational Therapy, Rheumatology, Orthopedics.

Autor para correspondência: Marilles Fuchs, Curso de Terapia Ocupacional, Departamento de Terapia Ocupacional, Universidade Federa do Paraná - UFPR, Rua Professor Lothario Meissner, 632, Bloco Didático II da Saúde, Jardim Botânico, CEP 80210-170, Curitiba, PR, Brasil, e-mail: mari.fuchs@hotmail.com 


\section{Introdução}

A dor é o motivo mais frequente de busca por serviços de saúde no Brasil, pois cerca de 70\% das pessoas atendidas apresentam a dor como queixa principal (TEIXEIRA et al., 2001; DE CARLO; QUEIROZ; SANTOS, 2007). De acordo com a Sociedade Brasileira para o Estudo da Dor, aproximadamente 50 milhóes de brasileiros apresentam dores crônicas e estima-se que de 15 a $25 \%$ dos adultos sofrerão de dor crônica em algum momento da vida (GARCIA, 2007b). Assim, a dor pode ser considerada um problema de Saúde Pública. Dada a abrangência numérica dos atendimentos cuja origem está relacionada com dor, torna-se relevante aprofundar estudos sobre as intervençóes realizadas pelo Terapeuta Ocupacional.

Para os pacientes com doenças reumáticas ou lesóes ortopédicas, a dor é um dos pontos centrais nas discussôes, sendo o fator negativo mais referido pelos sujeitos. É apontada como queixa principal de $98 \%$ das pessoas que são consultadas por reumatologistas (PAIVA et al., 2006).

O presente estudo irá abordar o problema dos sujeitos adultos que apresentam dor crônica decorrente de doenças ortopédicas e reumáticas - excluindo-se dor de origem neuropática, dor relacionada ao membro fantasma e dor oncológica - e teve como objetivo averiguar os tipos de procedimentos adotados pelos Terapeutas Ocupacionais brasileiros e do exterior, para intervir junto a pacientes com dor crônica.

A dor é considerada uma experiência individual desagradável, multidimensional, subjetiva, de alta complexidade e a resposta a esta varia de sujeito para sujeito - de acordo com a personalidade, o estado psicológico, as condições orgânicas, as experiências anteriores e o contexto sociocultural. Também envolve aspectos físicos, sensoperceptivos, psicológicos, emocionais, de aprendizado, éticos, ocupacionais, comportamentais, religiosos e morais (DE CARLO; QUEIROZ; SANTOS, 2007).

De acordo com a International Association for the Study of Pain (IASP), a dor é considerada uma

[...]experiência sensorial e emocional desagradável, que está relacionada a um dano tecidual, sendo este real ou potencial, ou descrita nos termos deste dano. (MERSKEY; BOGDUK, 2007).

A percepção da dor é influenciada por diversos fatores, como o estado biológico, o nível cultural, o meio social, a religião, os possíveis ganhos secundários, a manipulação, a atençáo e as experiências anteriores de dor (BEECHER, 1959², apud STANOS; HOULE, 2006; GARCIA, 2007a; ENGEL, 2005).

A dor, porém, é um processo natural, essencial à sobrevivência, um mecanismo de alerta que indica ao organismo a presença de dano tecidual para que esse reaja ativando os mecanismos de defesa, visando garantir sua proteção (CAILLIET, 1999). Se a dor torna-se crônica, esta deixa de ter a funçáo de alerta e passa a ser considerada desnecessária (SPECIALI; GONÇALVES, 2007), ou seja, patológica. A dor crônica gera repercussões biopsicossociais (MELLO et al., 2004), que podem produzir mudanças na personalidade, nos hábitos e rotinas de vida e na funcionalidade do sujeito (ENGEL, 2005). O bem-estar, a qualidade do sono, as interaçôes sociais, as reaçôes emocionais, a disposição e as habilidades físicas são comprometidas pela dor (NUNES, 2007) e o indivíduo tem sua rotina modificada e seu desempenho ocupacional prejudicado.

Nos últimos anos, houve um aumento da ocorrência de dor e diversas hipóteses apontam que essa elevaçáo pode ser decorrente dos novos hábitos de vida, das modificaçôes do meio ambiente, da maior longevidade dos indivíduos, em geral, e do aumento da sobrevida dos doentes que apresentam afecçôes fatais, da redução da tolerância do homem moderno ao sofrimento e do reconhecimento de novos conceitos que explicam as condiçóes álgicas (TEIXEIRA et al., 2001).

A dor crônica, por não ter função indicativa da iminência de um dano ao organismo, é considerada uma dor desnecessária (SPECIALI; GONÇALVES, 2007) e está presente mesmo após a eliminação do estímulo ou a cura do tecido lesado (MIRABELLISUSENS, 2004). Deixa de ser considerada aguda quando perdura por mais de seis meses e persiste além do tempo esperado para a cura da lesão. Geralmente, está associada a doenças crônicas, sendo na maioria das vezes o principal sintoma (FIGUEIRÓ, 2000); porém, a cronicidade da dor não é resultado de uma extensão do quadro agudo de dor (CAILLIET, 1999). A dor crônica não apresenta localização bem definida como a aguda e está relacionada aos componentes subjetivos de cada indivíduo (MIRABELLI-SUSENS, 2004). Pode ser possível demonstrar sua origem, porém a etiologia é muitas vezes indefinida (SPECIALI; GONÇALVES, 2007) e resultante de excesso de estimulação nociva ao corpo ou de alteraçóes no sistema nervoso central (FIGUEIRÓ, 2000).

As incapacidades decorrentes da dor crônica ocorrem em três níveis: funcional, social e laborativo 
(PENGO; SANTOS, 2004). Esse quadro gera repercussóes biopsicossociais, como já mencionado, afeta o desempenho ocupacional diário e os papéis ocupacionais do sujeito acometido, pois pode limitar sua participação nas tarefas ocupacionais diárias e interferir diretamente na vida desse sujeito e na qualidade da mesma (BRACCIANO; EARLEY, 2005; ENGEL, 2002, 2005; MELLO et al., 2004; RADOMSKI, 2005).

A dor apresenta linguagem particular; portanto, a sensação de dor é quantificada e qualificada pela palavra e o sentido que cada um dá a esta. Pode ser observada pelo comportamento, que reflete na capacidade funcional e nas açóes ocupacionais do sujeito (NUNES, 2007). Em razão da subjetividade da dor, ressalta-se a necessidade de utilizar-se da escuta do relato do paciente e também da observação de seu comportamento no processo de avaliação da dor. Assim, para uma avaliação adequada, é necessário que se utilizem instrumentos válidos e confiáveis (ENGEL, 2002), pois a padronização permite que a sensação subjetiva da dor seja expressa de forma mais objetiva pelo sujeito (BARROSO, 2005). Por outro lado, o instrumento utilizado deve ser de fácil aplicabilidade e possibilitar adaptaçáo para o nível de compreensão que o indivíduo apresenta (SANTANA, 2007).

Os instrumentos utilizados para mensuração da dor são, no geral, divididos em unidimensionais e multidimensionais (BARROSO, 2005; AIRES, 2007; SANTANA, 2007). As avaliaçôes unidimensionais avaliam apenas uma dimensão da experiência dolorosa; por outro lado, as multidimensionais consideram as diferentes qualidades que envolvem a dor, que incluem fatores fisiológicos, comportamentais, contextuais e autorregistros dos pacientes (SANTANA, 2007).

$\mathrm{Na}$ medida em que a dor impacta na qualidade de vida, é imprescindível - pela sua multidimensionalidade - que o sujeito acometido por dor crônica receba atendimento especializado por uma equipe multidisciplinar (DE CARLO; ELUI; PACKER, 2007), que pode ser composta por Terapeutas Ocupacionais, Médicos, Dentistas, Enfermeiros, Psicólogos, Fisioterapeutas, Assistentes Sociais e Nutricionistas (DE CARLO; QUEIROZ; SANTOS, 2007). O foco do Terapeuta Ocupacional é a melhora do desempenho ocupacional (PEDRETTI; EARLY, 2005) e seu papel é de promover uma vida com significado e produtiva, mesmo com a dor, por meio da prevenção de incapacidades, da promoção da função e do bem-estar no desempenho das atividades cotidianas (DE CARLO; ELUI; PACKER, 2007), do desenvolvimento das habilidades e capacidades físicas, da otimização das funções e dos hábitos ocupacionais que quebram o círculo vicioso dor-ociosidade-dor; proporciona-se ao sujeito, dessa forma, domínio de si mesmo e do ambiente, além da inserção social (DE CARLO et al., 2004; PENGO; SANTOS, 2004), com a manutenção e o resgate dos papéis ocupacionais.

Com a elaboração deste trabalho, objetivou-se responder ao problema que envolve o processo de Terapia Ocupacional junto a pacientes de Reumatologia e Ortopedia que apresentam quadro de dor crônica. Os objetivos específicos do presente trabalho foram: 1 - compreender o quadro clínico $\mathrm{da}$ dor bem como as suas consequências na vida do sujeito; 2 - verificar como o processo da dor interfere no desempenho ocupacional e reduz a qualidade de vida dos indivíduos; 3 - analisar métodos, protocolos e avaliações que são utilizados na mensuração da dor junto a pacientes com quadro álgico crônico; 4 - descrever os métodos de redução e tratamento da dor, e 5 - elucidar e discutir sobre as intervençóes da Terapia Ocupacional no tratamento da dor crônica.

\section{Metodologia}

Para a elaboraçáo deste trabalho, foi realizada revisão de literatura, a qual, segundo Boaventura (2004), tem como objetivos buscar o que já há de escrito sobre o tema que se pretende estudar, realizar análise e sintetizar as informaçóes, de modo a discutir e produzir novos dados sobre a temática, ao solucionar o problema ou ao levantar novos pontos que precisam ser pesquisados.

Como uma das etapas da revisão, realizou-se a pesquisa bibliográfica de referenciais teóricos nacionais e internacionais no acervo da Biblioteca de Ciências da Saúde da UFPR e nas bases de dados Lilacs, SciELO, Medline, OTseeker e Otdbase, escritos no período compreendido entre 1999 e 2009. Para tanto, foram utilizados os seguintes descritores de assunto: dor, terapia ocupacional, qualidade de vida, reabilitaçấo, reumatologia, ortopedia e medição da dor. Os descritores foram utilizados de forma associada, porém com obrigatoriedade do uso dos descritores: terapia ocupacional e dor. Além disso, realizou-se uma varredura de outros artigos escritos pelos autores que eram encontrados.

A seleção dos artigos científicos teve como base o seguinte critério de inclusão: trabalhos escritos por Terapeutas Ocupacionais brasileiros ou do exterior que abordam parte ou todo o processo de intervenção em Terapia Ocupacional com pacientes que apresentam dor crônica em decorrência de doenças ortopédicas ou reumáticas. Foram excluídos 
aqueles que tratavam de dor crônica de origem neuropática, dor relacionada a membro fantasma ou dor oncológica, além daqueles que citavam a atuação do Terapeuta Ocupacional, porém nenhum dos autores era Terapeuta Ocupacional. A verificação da profissão dos autores dos artigos foi realizada buscando-se a sua qualificação contida em nota de rodapé ou constatada durante a leitura do artigo na íntegra. A origem da dor da qual tratava o artigo era averiguada no próprio título ou após leitura de resumo ou do artigo na íntegra.

Durante a pesquisa bibliográfica, os artigos eram previamente selecionados. Inicialmente, selecionaram-se 23 artigos para análise mais criteriosa, incluindo leitura na íntegra. Com a conclusão dessa etapa, foram excluídos 15 artigos, restanto oito artigos, os quais foram utilizados neste trabalho.

\section{Resultados e discussão}

Para elaboração deste artigo, foram selecionados oito artigos, sendo seis brasileiros e dois estrangeiros, conforme a Tabela 1.

\section{A Terapia Ocupacional e a dor crônica}

O processo de Terapia Ocupacional é composto por procedimentos ditos gerais ou interdisciplinares, ou seja, comuns aos profissionais da área da saúde e que apresentam linguagem universal, e os específicos da profissão, que são caracterizados pela linguagem singular (CANÍGLIA, 2005). Os procedimentos gerais estão focados na saúde em geral e, dentre estes, se podem citar: diagnóstico, avaliação, entrevistas, encaminhamento, entre outros (CANÍGLIA, 2005).

$\mathrm{Na}$ Terapia Ocupacional, o processo engloba coleta de informaçôes sobre o paciente, a situação e os problemas; avaliaçáo dos dados coletados; definição das metas de intervenção; estabelecimento de prioridades; seleção da ação necessária; implementação da ação selecionada, e avaliação dos resultados obtidos (HAGEDORN, 2001).

\subsection{Avaliações da dor}

Há diversas escalas e meios de avaliação da dor de modo a tentar objetivar as características subjetivas que esta apresenta (YOSIPOVITCH; HUNDLEY, 2004³ apud SANTANA, 2007), já que é impossível mensurá-la por meio de instrumentos físicos. Por outro lado, é praticamente impossível que uma avaliação seja totalmente objetiva, uma vez que diversos fatores subjetivos são potenciais para alterar os resultados.

Nos artigos selecionados, foram identificados diferentes tipos de avaliaçóes da dor (Tabela 2). Há infinitas opçóes de mensuração da dor e, a cada nova publicação, os autores descrevem novas ferramentas para avaliar a dor (STRONG, 1999).

Tabela 1. Discriminação dos títulos, autores e ano de publicação dos artigos selecionados.

\begin{tabular}{|c|c|c|c|}
\hline & Autor(es) do artigo & Título do artigo & $\begin{array}{c}\text { Ano de } \\
\text { publicação }\end{array}$ \\
\hline Artigo 1 & STRONG, J. & Assessments of pain perception in clinical practice. & 1999 \\
\hline Artigo 2 & $\begin{array}{l}\text { MÜLLERSDORF, M.; } \\
\text { SÖDERBACK, I. }\end{array}$ & $\begin{array}{l}\text { Occupational therapist's assessments of adults with } \\
\text { long-term pain: The Swedish experience. }\end{array}$ & 2002 \\
\hline Artigo 3 & $\begin{array}{l}\text { NOORDHOEK, J.; } \\
\text { LOSCHIAVO, F. Q. }\end{array}$ & $\begin{array}{l}\text { Intervenção da Terapia Ocupacional no tratamento } \\
\text { de indivíduos com doenças reumáticas utilizando a } \\
\text { abordagem da proteção articular. }\end{array}$ & 2005 \\
\hline Artigo 4 & $\begin{array}{l}\text { NUNES, C. M. P.; } \\
\text { PENA, L. R. }\end{array}$ & $\begin{array}{l}\text { Atuação da Terapia Ocupacional no gerenciamento da } \\
\text { dor crônica para o trabalho no complexo do HC/UFMG. }\end{array}$ & 2007 \\
\hline Artigo 5 & $\begin{array}{l}\text { CARVALHO, M. G. R.; } \\
\text { NOORDHOEK, J.; } \\
\text { SILVA, M. C. O. }\end{array}$ & $\begin{array}{l}\text { Grupo de Orientação a indivíduos acometidos por } \\
\text { doenças reumáticas: espaço educativo e terapêutico. }\end{array}$ & 2006 \\
\hline Artigo 6 & $\begin{array}{l}\text { AGNOLON, M. C.; } \\
\text { SANTOS, S. S.; } \\
\text { ALMEIDA, M. H. M. }\end{array}$ & $\begin{array}{l}\text { Grupo de orientação postural a idosos com dor } \\
\text { osteomuscular: estabelecendo relações entre teoria e } \\
\text { prática. }\end{array}$ & 2006 \\
\hline Artigo 7 & TSUKIMOTO, G. R. et al. & $\begin{array}{l}\text { Avaliação longitudinal da escala de postura para dor } \\
\text { lombar crônica através da aplicação dos questionários } \\
\text { Roland Morris e Short Form Health Survey (SF-36). }\end{array}$ & 2006 \\
\hline Artigo 8 & $\begin{array}{l}\text { NOORDHOEK, J.; } \\
\text { JOKL, L. }\end{array}$ & $\begin{array}{l}\text { Efeito da música e de exercícios físicos num grupo de } \\
\text { pessoas reumáticas: estudo piloto. }\end{array}$ & 2008 \\
\hline
\end{tabular}


Tabela 2. Discriminação das avaliações mencionadas nos artigos.

\begin{tabular}{|c|c|c|}
\hline & Autor(es) & Avaliações \\
\hline Artigo 1 & STRONG, J. & $\begin{array}{l}\text { Avaliação para descrição da dor - intensidade, qualidade ou } \\
\text { localização - (Escala de avaliação numérica, escala analógica e } \\
\text { visual verbal, desenho da dor, Questionário de dor McGill), registro } \\
\text { de respostas a dor (Survey of Pain Attitudes Revised (SOPAR), } \\
\text { Pain Beliefs and Perceptions Inventory (PBPI), Tampa Scale for } \\
\text { Kinesiophobia, Fear-Avoidance Beliefs Questionnaire, observação } \\
\text { clínica da resposta a dor) e impacto da dor na vida (autorrelato, } \\
\text { diários de atividades, Oswestry Low Back Pain Disability } \\
\text { Questionnaire (OLBPDQ), Pain Disability Index (PDI), Sickness } \\
\text { Impact Profiles (SIP)). }\end{array}$ \\
\hline Artigo 2 & $\begin{array}{l}\text { MÜLLERSDORF, M.; } \\
\text { SÖDERBACK, I. }\end{array}$ & Occupational Therapy Needs Assessment - Pain (OTNA - P) \\
\hline Artigo 3 & $\begin{array}{l}\text { NOORDHOEK, J.; } \\
\text { LOSCHIAVO, F. Q. }\end{array}$ & Não cita. \\
\hline Artigo 4 & $\begin{array}{l}\text { NUNES, C. M. P.; } \\
\text { PENA, L. R. }\end{array}$ & Não cita. \\
\hline Artigo 5 & $\begin{array}{l}\text { CARVALHO, M. G. R.; } \\
\text { NOORDHOEK, J.; } \\
\text { SILVA, M. C. O. }\end{array}$ & $\begin{array}{l}\text { Cita a utilização de avaliações qualitativas para identificar grau de } \\
\text { satisfação do paciente com relação ao grupo, porém não cita quais. }\end{array}$ \\
\hline Artigo 6 & $\begin{array}{l}\text { AGNOLON, M. C.; } \\
\text { SANTOS, S. S.; } \\
\text { ALMEIDA, M. H. M. }\end{array}$ & $\begin{array}{l}\text { Formulário sobre presença e grau de dor nas atividades cotidianas, } \\
\text { por meio de entrevista individual, porém não cita qual a estrutura } \\
\text { e se esta é padronizada. Faz um comparativo entre a dor em sete } \\
\text { atividades cotidianas antes e após os atendimentos ("dor reduzida", } \\
\text { "sem dor", "dor inalterada"). }\end{array}$ \\
\hline Artigo 7 & TSUKIMOTO, G. R. et al. & $\begin{array}{l}\text { Escala Visual Analógica (EVA), Short Form Health Survey (SF-36) } \\
\text { e Rolando-Morris (RM). }\end{array}$ \\
\hline Artigo 8 & $\begin{array}{l}\text { NOORDHOEK, J.; } \\
\text { JOKL, L. }\end{array}$ & $\begin{array}{l}\text { Entrevista quanto ao estado emocional e físico -intensidade da dor e } \\
\text { facilidade de se movimentar. }\end{array}$ \\
\hline
\end{tabular}

A seleção da ferramenta de avaliação irá depender do tipo de paciente atendido e, para que seja confiável, deve-se levar em conta o contexto específico (STRONG, 1999), adequando-se ao nível de compreensão do sujeito e da facilidade na aplicação (SANTANA, 2007). A escolha de uma avaliação da dor que seja apropriada e precisa não é tarefa fácil, em decorrência da já mencionada subjetividade que permeia o quadro álgico (McCAHON et al., 2005).

A avaliaçáo da dor apresenta três componentes essenciais: descrição da dor, registro das respostas à dor e a dimensão do impacto causado na vida do sujeito, que são subdivididos em categorias, ou seja, há diversas ferramentas ou estilos de avaliação para cada item (STRONG, 1999).

Em consonância com o que já foi relatado, muitos autores afirmam que as avaliaçôes são complementares e que, para verificar a eficiência e a resposta ao tratamento, é importante aplicar as avaliaçóes no início, durante o transcorrer e ao fim do tratamento (TSUKIMOTO et al., 2006; STRONG, 1999).

O aprofundamento da avaliaçáo da dor vai depender de outros fatores contextuais, como o tempo disponível, o local de atendimento e as características de precipitação da dor.

O ideal seria o monitoramento da dor, como já pontuado, comparando sua intensidade pouco antes do início do tratamento, durante e ao término deste, além de que as escalas lineares permitiriam essa comparação de mudanças (STRONG, 1999). Por outro lado, quando o paciente apresenta uma dor de complexa e difícil localização ou compreensão, a utilizaçáo apenas da medida de intensidade da dor torna-se inadequada; entáo, é necessária uma avaliação mais completa, como, por exemplo, a avaliação das respostas à dor e do impacto da mesma na vida do sujeito.

Se a atenção do tratamento é voltada para múltiplos aspectos do dia a dia e ao ensino de formas de controlar os sintomas que interferem nesse cotidiano, faz-se necessário aplicar não somente uma avaliação voltada à queixa dolorosa, mas a todos os outros desfechos objetivados com o tratamento (TSUKIMOTO et al., 2006).

As avaliações que descrevem a dor podem considerar a intensidade, a qualidade ou a localização 
da dor no corpo e, geralmente, apresentam o estilo de autorrelato, como questionários, escalas de classificação, escalas analógicas visuais e desenhos. Dentre essas avaliações, os artigos selecionados citam: a escala de categoria numérica, a escala visual analógica (EVA), o desenho da dor e o Questionário de Dor McGill. A escala de categoria numérica é vista como a mais popular, apesar de a escala visual analógica e a escala da avaliação verbal também serem bastante utilizadas (STRONG, 1999).

Provavelmente, em decorrência das facilidades apresentadas pelas escalas unidimensionais - em especial, a Escala Visual Analógica e a Escala de Categoria Numérica -, como a simples aplicabilidade, a não necessidade de treinamento e a resposta objetiva e pontual, estas tenham sido as avaliaçóes mais citadas pelos autores. Por outro lado, apesar de os instrumentos de mensuração unidimensional da dor serem os mais utilizados, estes tornam a experiência dolorosa demasiadamente simplificada (SANTANA, 2007).

Quanto às respostas à dor, estas são individualizadas e variam de sujeito para sujeito, de acordo com características pessoais, experiências anteriores e ambiente, demonstradas por meio de reaçôes e mudanças psicológicas ou comportamentais (STRONG, 1999; DE CARLO; QUEIROZ; SANTOS, 2007). O medo do sujeito, a crença sobre a causa da dor ou a expectativa de recorrência da dor pode interferir no tratamento e na recuperação do sujeito (STRONG, 1999).

As avaliaçóes da resposta à dor encontradas foram The Survey of Pain Atitudes - Revised (SOPAR), Pain Beliefs and Perceptions Inventory (PBPI) e a observação clínica das respostas à dor. Além disso, ressalta-se que seria interessante avaliar o medo do movimento e a crença da reincidência da dor por meio de outras escalas, como Tampa Scale for Kinesiophobia e Fear-Avoidance Beliefs Questionnaire (STRONG, 1999).

A observação clínica também é citada como meio de avaliação e complemento da avaliação formal. Os autores relatam que esta pode ser usada durante o envolvimento do paciente nas atividades de avaliação e tratamento, e observada por meio de expressóes faciais ou reaçôes de proteção ou tensão de regiôes do corpo. Nesse caso, deve-se tomar cuidado com as falsas expressôes de dor que os indivíduos podem realizar (STRONG, 1999).

Outro aspecto citado que deve ser considerado no processo de avaliação é o impacto que a dor causa na vida do sujeito e de seus familiares e cuidadores, que pode ser avaliado de diversas formas, como por meio de questionários, autorrelatos e diários de dor, dependendo do contexto (STRONG, 1999). Os autores pesquisados que abordam o impacto da dor citam as seguintes avaliaçóes: Oswestry Low Back Pain Desability Questionnaire (OLBPDQ), Pain Disability Index (PDI), Sickness Impact Profile (SIP) e diários de atividades (STRONG, 1999).

Ainda em se tratando da interferência da dor na vida do sujeito, Tsukimoto et al. (2006) citam o Questionário Roland-Morris e especificam que este é uma avaliação da incapacidade voltada para a análise dos impactos na funcionalidade do sujeito causados pela lombalgia, sem, entretanto, enfatizar os aspectos psicossociais que podem envolvê-la (TSUKIMOTO et al., 2006). Esse instrumento é baseado no Sickness Impact Profile (SIP). Além destes, também foi apontado o instrumento de avaliação Short Form Health Survey (SF-36), um questionário genérico de qualidade de vida, multidimensional, dividido em oito domínios avaliados separadamente. Um desses domínios é a dor, com dois itens, que mensuram a intensidade da dor em seis níveis, objetivando avaliar qual a influência da dor nas atividades da vida diária (TSUKIMOTO et al., 2006).

No Artigo 6, constatou-se a utilização de entrevista (inicial e ao término dos atendimentos) como meio de avaliação e acompanhamento do quadro; porém, não foi possível perceber a utilização de um protocolo padronizado para esse levantamento (AGNOLON; SANTOS; ALMEIDA, 2006).

No Artigo 2, os autores objetivaram descrever, analisar e classificar qual a relação entre as necessidades de intervenção de Terapia Ocupacional que o sujeito com dor crônica apresenta e quais as sugestóes de intervençóes geralmente utilizadas pelos Terapeutas Ocupacionais para atender a essas necessidades. Para tanto, os autores trazem o instrumento de avaliação OTNA-P (Occupational Therapy Needs Assessment - Pain), dividido em 18 itens, os quais têm como objetivos determinar a localizaçáo da dor e avaliar qualitativamente a principal limitação de atividade, além de outros itens baseados nas consideraçôes relacionadas às áreas, aos objetivos e às intervençôes realizadas pelos Terapeutas Ocupacionais, identificadas após revisão de literatura. Apesar de essa avaliação ser destinada a pacientes com dor crônica de origem musculoesquelética, esta avalia não apenas a dor, mas sua interferência na vida do sujeito (MÜLLERSDORF; SÖDERBACK, 2002).

Ao considerar a avaliação como essencial para o andamento do Processo de Terapia Ocupacional, ressalta-se a importância de os pacientes acometidos por quadro crônico de dor serem avaliados pelo 
Terapeuta Ocupacional, de modo a reconhecer qual a intensidade da dor, como o sujeito reage a essa dor e qual a interferência no desempenho de suas atividades cotidianas.

O Terapeuta Ocupacional pode utilizar as avaliações citadas neste trabalho que não são específicas da Terapia Ocupacional, mas que fornecem informaçóes relevantes para sua atuação. Outros dados podem ser obtidos por meio da entrevista inicial, bem como da observação clínica (CARVALHO; NOORDHOEK; SILVA, 2006; AGNOLON; SANTOS; ALMEIDA, 2006; NOORDHOEK; JOKL, 2008).

Das avaliações citadas neste artigo, não são todas que têm tradução, adaptação cultural e validação para a língua portuguesa. As avaliaçóes validadas no Brasil são: Escala de Categoria Numérica, Escala Visual Analógica (EVA), Desenho da Dor, Questionário de Dor McGill, The Survey of Pain Atitudes - Revised (SOPAR), Tampa Scale for Kinesiophobia, FearAvoidance Beliefs Questionnaire, Questionário Roland-Morris e Short Form Health Survey (SF-36).

\subsection{Objetivos de intervenção do Terapeuta Ocupacional}

A definição de objetivos de tratamento é considerada a próxima etapa do processo de intervenção do Terapeuta Ocupacional. Nesta pesquisa, foram encontrados diversos objetivos para intervenção junto a pacientes com dor crônica (Tabela 3).

Destacam-se os objetivos gerais da profissão não voltados apenas ao tratamento da dor, que foram mencionados: maximizar o controle do sujeito sobre sua vida e seu nível funcional, e minimizar a perda de papéis e as competências relacionadas a esses papéis (MÜLLERSDORF; SÖDERBACK, 2002). Os objetivos de intervenção do Terapeuta Ocupacional voltados à dor crônica citados são: reduzir a dor, promover saúde, melhorar desempenho na manutenção da casa, melhorar postura e mecânica corporal, melhorar qualidade de vida, aumentar independência, aumentar responsabilidade por si, aumentar controle da dor, aumentar tolerância à

Tabela 3. Discriminação dos objetivos de intervenção da Terapia Ocupacional mencionados nos artigos.

\begin{tabular}{lll}
\hline \multicolumn{1}{c}{ Autor(es) } & \multicolumn{1}{c}{ Objetivos de intervenção. } \\
\hline Artigo 1 & STRONG, J. & Não cita, por não ser o foco. \\
\hline Artigo 2 & MÜLLERSDORF, M.; & Avaliar necessidade de intervenção da Terapia Ocupacional. Na \\
& SÖDERBACK, I. & revisão, encontrou como objetivos: reduzir a dor, promoção de \\
& saúde, melhorar desempenho na manutenção da casa, melhorar \\
& postura e mecânica corporal, melhorar qualidade de vida, \\
& maximizar a independência, maximizar autorresponsabilidade, \\
& maximizar controle da dor, aumentar tolerância a dor, elevar \\
& autoestima, manter/restabelecer competência, manter/restabelecer \\
& papéis, maximizar função, recuperar equilíbrio nas atividades \\
& cotidianas, recuperar controle, restaurar autoeficácia.
\end{tabular}

\begin{tabular}{|c|c|c|}
\hline Artigo 3 & $\begin{array}{l}\text { NOORDHOEK, J.; } \\
\text { LOSCHIAVO, F. Q. }\end{array}$ & $\begin{array}{l}\text { Esclarecer e apontar possibilidades de gerenciar e controlar a } \\
\text { dor; evitar a dor e o estresse articular na execução das atividades } \\
\text { diárias. }\end{array}$ \\
\hline Artigo 4 & $\begin{array}{l}\text { NUNES, C. M. P.; } \\
\text { PENA, L. R. }\end{array}$ & $\begin{array}{l}\text { Controle da dor crônica e mudança de hábito nas atividades } \\
\text { de vida diária, no trabalho e atividades produtivas. Promover } \\
\text { consciência corporal. }\end{array}$ \\
\hline Artigo 5 & $\begin{array}{l}\text { CARVALHO, M. G. R.; } \\
\text { NOORDHOEK, J.; } \\
\text { SILVA, M. C. O. }\end{array}$ & $\begin{array}{l}\text { Educação e suporte do paciente. Promover habilidade de } \\
\text { desempenho em atividades diárias, prevenir perdas funcionais e } \\
\text { propor adaptações adequadas. Socialização, desenvolvimento de } \\
\text { habilidades pessoais, novas possibilidades de enfrentamento da } \\
\text { doença e melhorar desempenho de papéis ocupacionais. }\end{array}$ \\
\hline Artigo 6 & $\begin{array}{l}\text { AGNOLON, M. C.; } \\
\text { SANTOS, S. S.; } \\
\text { ALMEIDA, M. H. M. }\end{array}$ & $\begin{array}{l}\text { Redução da dor nas atividades cotidianas, estimulação e adoção de } \\
\text { habilidades de autocuidado. Melhora da qualidade de vida. }\end{array}$ \\
\hline Artigo 7 & TSUKIMOTO, G. R. et al. & $\begin{array}{l}\text { Educação para os cuidados básicos de prevenção e redução de } \\
\text { episódios de dor. }\end{array}$ \\
\hline Artigo 8 & $\begin{array}{l}\text { NOORDHOEK, J.; } \\
\text { JOKL, L. }\end{array}$ & $\begin{array}{l}\text { Esclarecimento de dúvidas sobre a moléstia e seu tratamento, } \\
\text { fornecendo meios que auxiliem na superação dos desafios } \\
\text { impostos pela moléstia. }\end{array}$ \\
\hline
\end{tabular}


dor, aumentar autoestima, manter e/ou restabelecer competência, manter e/ou restabelecer papéis, maximizar função, recuperar equilíbrio nas atividades diárias, recuperar controle e restaurar autoeficácia (MÜLLERSDORF; SÖDERBACK, 2002). Ressalta-se que os objetivos que foram mais citados nos artigos não são exclusivos para o tratamento da dor, mas gerais da profissão, como se pode exemplificar: aumentar independência, aumentar autoestima, manter e/ou restabelecer competência, maximizar função, manter e/ou restabelecer papéis e recuperar controle.

Também foi encontrado como objetivo de intervenção em Terapia Ocupacional a educação do sujeito com relação à prevenção e à redução da dor, para as quais é necessária a aquisição e/ ou a mudança de hábitos de vida, o que incentiva o sujeito a responsabilizar-se por seu tratamento e recuperação (TSUKIMOTO et al., 2006). No Artigo 4, os autores pontuam como objetivos em Terapia Ocupacional o controle da dor e a mudança de hábitos nas atividades cotidianas - vida diária e trabalho, e atividades produtivas - que, de certo modo, interferem no controle da dor (NUNES; PENA, 2005).

Em contraponto com alguns autores, há aqueles que apontam que o real objetivo da intervenção na dor não é necessariamente minimizar a intensidade desta, mas sim promover a máxima função, independentemente da existência da dor, com a mudança de hábitos visando minimizar a dor durante a execução das atividades cotidianas como o fator crucial além da redução da dor (McCAHON et al., 2005; AGNOLON; SANTOS; ALMEIDA, 2006).

Portanto, percebe-se que os objetivos apontados pelos autores visam além de gerenciar e controlar a dor, também retomar e/ou modificar esses aspectos - como hábitos, rotinas, bem-estar, sono, socializaçâo e habilidades físicas - de modo a refletir na melhora do desempenho ocupacional do sujeito e, consequentemente, na qualidade de vida do mesmo.

Com exceção do Artigo 1, todos os outros abordam ao menos um objetivo de intervenção. Por outro lado, muitos dos autores dos artigos selecionados não deixam claro quais objetivos estão voltados para a intervenção junto a pacientes com problemas ortopédicos e reumáticos que apresentam quadro de dor crônica - delimitado neste trabalho - ou para os problemas ortopédicos e reumáticos de um modo geral, foco este de muitos dos artigos selecionados.

\subsection{Modalidades de atendimento}

As modalidades de atendimento pelas quais o Terapeuta Ocupacional pode alcançar seus objetivos são: modalidade de atendimento grupal e de atendimento individual.

A ideia de que o fazer humano tem potencial terapêutico é um dos fundamentos que norteiam a prática do Terapeuta Ocupacional. No grupo, os integrantes se deparam com

[...] a possibilidade de experimentar outras formas de se relacionar e de vivenciar situaçóes inéditas relativas ao fazer, possibilitando que a ação ganhe um sentido e um significado. (BALLARIN, 2007, p. 40).

Portanto, o grupo proporciona o meio pelo qual o sujeito pode apresentar e discutir sobre seus problemas, e buscar formas alternativas de desempenhar suas atividades, de acordo com sua necessidade, prioridade e vontade. A intervenção em grupos é considerada apropriada quando se trata de pacientes que apresentam doenças crônicas (NORDENSKIÖLD; ALTHOFF; HANSEN, $1994^{4}$ apud NOORDHOEK; LOSCHIAVO, 2005).

O Artigo 6 aponta que o grupo exerce papel de facilitador da participação dos sujeitos e que seria maior o compartilhamento da experiência da dor, bem como o compartilhamento das alternativas utilizadas para a reduçáo da mesma seria mais intenso em grupo; com isso, as repercussōes no cotidiano do sujeito seriam mais positivas (AGNOLON; SANTOS; ALMEIDA, 2006).

Dos artigos selecionados, cinco explicitam a modalidade de atendimento grupal como o meio de intervenção. Dois desses cinco também tratam da modalidade de atendimento individual (Tabela 4).

\subsection{Recursos terapêuticos}

Os recursos terapêuticos utilizados para alcançar os objetivos que envolvem o problema da dor crônica são bastante diversos e dependem de cada caso, do contexto de atendimento, da experiência do terapeuta, dentre outros fatores (Tabela 5).

O Artigo 2 descreve que há diversos recursos de tratamento utilizados por Terapeutas Ocupacionais, como treino de atividades da vida diária, dispositivo assistivo, simplificação da tarefa, aconselhamento, treino de relaxamento, instrução da mecânica corporal, órteses, controle do estresse, treino vocacional, atividades individuais e grupais de diversão, treino da tolerância ao trabalho e terapia 
Tabela 4. Discriminação das modalidades de atendimento mencionadas nos artigos.

\begin{tabular}{lll}
\hline & \multicolumn{1}{c}{ Autor(es) } & Modalidades de atendimento \\
\hline Artigo 1 & STRONG, J. & Não cita. \\
Artigo 2 & MÜLLERSDORF, M.; SÖDERBACK, I. & Não cita. \\
Artigo 3 & NOORDHOEK, J.; LOSCHIAVO, F. Q. & Individual e grupal. \\
Artigo 4 & NUNES, C. M. P.; PENA, L. R. & Individual e grupal. \\
Artigo 5 & CARVALHO, M. G. R.; NOORDHOEK, J.; SILVA, M. C. O. & Grupal. \\
Artigo 6 & AGNOLON, M. C.; SANTOS, S. S.; ALMEIDA, M. H. M. & Grupal. \\
Artigo 7 & TSUKIMOTO, G. R. et al. & Não deixa claro. \\
Artigo 8 & NOORDHOEK, J.; JOKL, L. & Grupal. \\
\hline
\end{tabular}

Tabela 5. Discriminação dos recursos terapêuticos mencionados nos artigos.

\begin{tabular}{|c|c|c|}
\hline & Autor(es) & Recursos terapêuticos \\
\hline Artigo 1 & STRONG, J. & Não cita, por não ser o foco. \\
\hline Artigo 2 & $\begin{array}{l}\text { MÜLLERSDORF, M.; } \\
\text { SÖDERBACK, I. }\end{array}$ & $\begin{array}{l}\text { Treino de AVD’s, tecnologia assistiva, simplificação de tarefa, } \\
\text { orientações, treino técnicas de relaxamento, instrução dos } \\
\text { mecanismos corporais, órteses, manejo do stress, treino vocacional, } \\
\text { atividades de lazer individual e grupal, treino de tolerância ao } \\
\text { trabalho, terapia familiar, conservação de energia, proteção } \\
\text { articular, ergonomia. }\end{array}$ \\
\hline
\end{tabular}

\begin{tabular}{ll}
\hline Artigo 3 & NOORDHOEK, J.; \\
& LOSCHIAVO, F, Q.
\end{tabular}

Artigo 4 NUNES, C. M. P.;
PENA, L. R.
Redimensionamento do cotidiano usando o método cognitivo- comportamental - utiliza recursos educacionais, ajuste de expectativas, modificação de crenças disfuncionais e medos infundados.
Relaxamento fisiológico; dessensibilização tátil para auxiliar na consciência corporal; cinesioterapia; jogos de motivação e expressão com incentivo à discussão de temas que envolvem o quadro álgico; demonstração de novas estratégias para desempenho ocupacional.

\begin{tabular}{lll}
\hline Artigo 5 & CARVALHO, M. G. R.; & Princípios de conservação de energia, técnicas de simplificação do \\
& $\begin{array}{l}\text { NOORDHOEK, J.; } \\
\text { SILVA, M. C. O. }\end{array}$ & $\begin{array}{l}\text { trabalho, programa de exercícios e proteção articular. Exercícios } \\
\text { de relaxamento e alongamento; atividades de socialização e lazer; } \\
\text { palestras interativas e interdisciplinares. }\end{array}$ \\
\hline
\end{tabular}

Artigo 6 AGNOLON, M. C.; $\quad$ Observação do desempenho e demonstração de forma alternativa; SANTOS, S. S.; $\quad$ massagem para prevenção e alívio da dor. Utiliza os princípios ALMEIDA, M. H. M. da conservação de energia, proteção articular, ergonomia e cinesiologia.

Artigo 7 TSUKIMOTO, G. R. et al. Aulas; orientações (reeducação alimentar, alongamentos, exercícios e cuidados com o corpo, atividades aeróbicas, uso de aparelhos em academias, aspectos ergonômicos relacionados às atividades de vida diária, do trabalho e lazer), sensibilização quanto às possibilidades de participação social.

Artigo 8 NOORDHOEK, J.; Discussões sobre proteção articular, manutenção de energia, JOKL, L. tratamento medicamentoso, nutrição e hábito alimentares, técnicas de relaxamento e exercícios físicos, recursos musicoterápicos.

familiar (STRONG, $1986^{5}$ apud MÜLLERSDORF; SÖDERBACK, 2002).

No Artigo 2, os autores agruparam as intervençôes voltadas para o tratamento da dor em seis categorias: educação e controle do estresse; intervençôes comportamentais; tratamento da mão; atividade de grupo; tolerância de atividade, e adaptação externa. Cada uma desstas categorias apresenta diversos recursos; assim, o item educação e controle do estresse engloba a definição de objetivos entre paciente e terapeuta, educação sobre a dor, modificação das atitudes em relação à dor, avaliação das atitudes em 
relação à dor, técnicas de relaxamento, controle do estresse, uso de biofeedback e comunicação da dor; o item intervençóes comportamentais discrimina aconselhamento, conservação de energia, ergonomia, treino de mecânica corporal e trabalho condicionado; a categoria tratamento de mão inclui órtese, proteção articular e educação; na atividade de grupo, tem-se grupo de atividades/aconselhamento e retorno à escola; o item tolerância de atividade engloba uso de atividades intencionais, tolerância/resistência à atividade e uso de artes e ofícios; no tópico adaptação externa, foram encontrados a adaptação do ambiente e os dispositivos assistivos/simplificação das atividades diárias (MÜLLERSDORF; SÖDERBACK, 2002).

Alguns pacientes com dor podem adotar posturas que resultam em dor tolerável ou mínima - mais confortáveis - denominadas antálgicas; porém, essas posturas podem prejudicar a função musculoesquelética. Portanto, o Terapeuta Ocupacional deve orientar o sujeito quanto às posturas durante a realização de suas atividades cotidianas - sentadas ou em pé (DE CARLO; ELUI; PACKER, 2007).

Nos artigos selecionados, dentre os principais recursos descritos pelos autores, estão as medidas protetoras que envolvem mudança de hábitos, como os princípios de proteção articular e conservação de energia. Segundo Palmer e Simons $\left(1991^{6}\right.$, apud TORQUETTI et al., 2008), a proteção articular é uma estratégia pela qual o indivíduo se autogerencia de modo a promover náo somente a redução da dor, como também para reduzir a fadiga e manter a integridade articular e a função. Segundo Hammond (19947 , apud TORQUETTI et al., 2008), proteção articular envolve concepçóes que englobam a conservação de energia, o uso de tecnologia assistiva e a alteração de padrões de movimento articular, além de respeitar a dor, oferecer descanso às articulaçóes e realizar exercícios para manutenção da amplitude de movimento e da força muscular.

Em consonância com o que foi apresentado, dentre as técnicas utilizadas e os princípios considerados para prevenir a ocorrência e reduzir a intensidade da dor, Agnolon, Santos e Almeida (2006) ressaltam a conservação de energia e a proteção articular também citadas por Noordhoek e Loschiavo (2005) -, como também a ergonomia e a cinesiologia. Esses autores, no Artigo 6, apontam a necessidade de observação do desempenho funcional dos pacientes e de demonstraçóes, tanto pelos pacientes como pelos profissionais, para propor alternativas de executar atividades realizadas com dor, também sugerindo outros recursos que previnem e aliviam a dor, como a massagem. Carvalho, Noordehoek e Silva
(2006) também apontam as técnicas de conservação de energia, simplificação de tarefa, programa de exercícios e proteção articular (Artigo 5).

Müllersdorf e Söderback (2002) encontraram a utilização da ergonomia para a intervenção junto à dor crônica (Artigo 2). Agnolon, Santos e Almeida (2006) e Tsukimoto et al. (2006) relacionam a utilização de princípios da ergonomia para a realização das atividades de vida diária (Artigo 7).

Nunes e Pena (2005) no Artigo 4, Müllersdorf e Söderback (2002) no Artigo 2, Carvalho, Noordhoek e Silva (2006, p. 135) no Artigo 5 e Noordhoek e Jokl (2008) no Artigo 8 citam que utilizaram técnicas de relaxamento como recurso terapêutico para o tratamento da dor. Nunes e Pena (2005) apontam que a dor é um distúrbio de excitação e que o relaxamento promove uma condição antagônica - favorece o estado de hipoexcitabilidade. Segundo De Carlo, Elui e Packer (2007), o relaxamento é um recurso terapêutico que pode ser aplicado pelos Terapeutas Ocupacionais. Este auxilia nos estados de estresse, tensão muscular ou como meio revigorante da saúde física, mental e emocional.

Apesar de não utilizarem os mesmos termos, observou-se que sete dos oito Artigos utilizaram no processo de intervenção do paciente os princípios de proteção articular, conservação de energia, simplificação do trabalho, ergonomia, cinesiologia e as técnicas de relaxamento.

Müllersdorf e Söderback (2002) apontaram o uso de tecnologia assistiva como recurso para tratamento da dor crônica. Segundo De Carlo, Elui e Packer (2007), a tecnologia assistiva, na qual se encontram as órteses, pode auxiliar ou compensar uma função e, assim, facilitar o desempenho das atividades cotidianas.

Outro recurso utilizado para a intervenção citado pelos autores é a educação do paciente. Esta pode ser feita de diversas formas e abordando diferentes conteúdos. Tsukimoto et al. (2006) propuseram que a educação ao paciente fosse feita por meio de aulas teóricas e práticas, englobando aquisição de conhecimento referente à patologia, orientaçóes relacionadas a alongamentos e exercícios, hábitos alimentares, ergonomia relacionada às atividades cotidianas e discussão sobre as possibilidades de participação social. Noordhooek e Jokl (2008) utilizam-se do mesmo modelo de educação ao paciente - além de discussão sobre técnicas de proteção articular e conservação de energia associado ao tratamento medicamentoso, à utilizaçáo de técnicas de relaxamento, aos exercícios físicos e aos recursos musicoterápicos. 
O comportamento do sujeito frente à doença pode ter relação íntima com a compreensão da mesma; dessa forma, o sujeito não percebe que seu comportamento pode influenciar no curso da doença ou mesmo não sabe como este pode ser modificado de modo a contribuir para sua recuperação (POLLEY; HUNDER, 1980 apud TORQUETTI et al., 2008). Assim, ressalta-se a importância da educação do paciente com relação à doença e às formas de modificaçáo do comportamento de modo a ele, paciente, assumir papel ativo perante sua vida.

\section{Considerações finais}

A temática dor, em especial a dor crônica, tem sido abordada de forma crescente no contexto da saúde por ser uma sensação desagradável e indesejada, que acarreta perda de desempenho funcional e papéis. Tais desdobramentos são combatidos pelos profissionais, visto que estes objetivam a máxima qualidade de vida possível ao sujeito e a dor assume papel impeditivo a tal objetivo. Além disso, o aumento da expectativa de vida e, com isso, um aumento mundial de pessoas com doenças crônicas (CARVALHO; NOORDHOEK; SILVA, 2006), associado à dor crônica, conduzem a uma busca de métodos para reduçâoo ou eliminação da mesma, ou ainda, a manutenção da máxima qualidade de vida apesar da presença da dor.

Com a elaboração deste trabalho, foi possível compreender as diversas dimensóes do quadro clínico da dor, bem como suas consequências no desempenho ocupacional e na qualidade de vida do sujeito. Além disso, distinguiram-se quais as ferramentas de mensuração da dor são utilizadas e quais as mais frequentes no caso da dor crônica. Possibilitou, ainda, conhecer quais os objetivos, as modalidades de atendimento e os recursos utilizados por Terapeutas Ocupacionais no tratamento da dor crônica.

Foi possível constatar que a dor necessita de intervenção multidisciplinar em razão da sua multidimensionalidade e pelo fato de afetar a vida do sujeito e o seu desempenho ocupacional diário. Entre os profissionais desta equipe, o Terapeuta Ocupacional é um importante componente, com papel fundamental, uma vez que o foco de sua intervenção engloba o máximo desempenho com autonomia e independência - nas atividades cotidianas, desempenho este que se encontra comprometido em decorrência do quadro álgico.

Também se constatou que as avaliaçóes mais utilizadas são as unidimensionais, dentre as quais a Escala de Categoria Numérica e a Escala Visual Analógica, por serem de aplicação rápida e sim bem de não precisar de treinamento.

Já entre as abordagens de tratamento utilizadas, a mais apontada pelos autores é a abordagem grupal e, entre os recursos terapêuticos encontrados, é a educação do paciente, que visa o controle da dor e não somente sua redução, a mais citada.

Com base nas ideias discutidas anteriormente, deve-se considerar que não há apenas um recurso terapêutico que possa ser utilizado com pacientes que apresentam determinado tipo de dor, sendo necessário considerar a necessidade do sujeito - sua individualidade, sua rotina, seus papéis ocupacionais, as atividades que exerce/ deseja exercer, suas potencialidades e limitaçôes, entre outras características individuais. Portanto, independentemente do recurso que se utilize, deve-se atentar para a forma de realizaçấo, as necessidades do sujeito, as possibilidades de desempenho e adaptação, as etapas exigidas, as habilidades necessárias, entre outros aspectos. Além disso, o Terapeuta Ocupacional deve se atentar que a dor envolve não apenas fatores físicos, mas também pessoais, psicossociais e culturais; dessa forma, aponta-se para uma intervenção voltada náo apenas para o controle da dor, mas sim para a promoçáo de mudança dos hábitos que desencadeiam o quadro de dor.

Com base nisso, pode-se afirmar que o sucesso da intervençāo depende de o sujeito ter papel ativo frente ao tratamento e de o Terapeuta Ocupacional assumir o papel de facilitador do desempenho ocupacional satisfatório e, assim, da melhor qualidade de vida do sujeito que apresenta dor crônica em decorrência de doenças ortopédicas e reumáticas.

\section{Referências}

AGNOLON, M. C.; SANTOS, S. S.; ALMEIDA, M. H. M. Grupo de orientaçáo postural a idosos com dor osteomuscular: estabelecendo relaçóes entre teoria e prática. Revista de Terapia Ocupacional da Universidade de São Paulo, v. 17, n. 2, p. 80-86, 2006.

AIRES, E. M. Características gerais do processo de avaliação. In: DE CARLO, M. M. R. P.; QUEIROZ, M. E. Dor e cuidados paliativos: Terapia Ocupacional e interdisciplinariedade. São Paulo: Roca, 2007. p. 63-69.

BALLARIN, M. L. G. S. Abordagens Grupais. In: CAVALCANTI, A.; GALVĀO, C. Terapia Ocupacional: fundamentação e prática. Rio de Janeiro: GuanabaraKoogan, 2007. p. 38-43.

BARROSO, P. N. Dor. In: SOCIEDADE BRASILEIRA DE TERAPEUTAS DA MÃO E MEMBRO SUPERIOR. Recomendaçôes para avaliação do membro superior. 2. ed. Joinville: SBTM, 2005. p. 30-37. 
BOAVENTURA, E. M. Revisão da Literatura. In: BOAVENTURA, E. M. Metodologia da Pesquisa: monografia, dissertação e tese. São Paulo: Atlas, 2004. p. 45-53.

BRACCIANO, A. G.; EARLEY, D. Modalidades de agentes físicos. In: TROMBLY, C. A.; RADOMSKI, M. V. Terapia Ocupacional para disfunçöes físicas. 5. ed. Sáo Paulo: Santos, 2005. p. 421-441.

CAILliET, R. Dor: mecanismos e tratamento. Porto Alegre: Artmed, 1999.

CANÍGLIA, M. Terapia Ocupacional: objeto e metodologia. Belo Horizonte: Expressa Artes gráficas, 1994. CANÍGLIA, M. Processo Terapêutico Ocupacional. In: CANÍGLIA, M. Terapia Ocupacional: um enfoque disciplinar. Belo Horizonte: Ophicina Arte e Prosa, 2005. p. 116-171.

CARVALHO, M. G. R.; NOORDHOEK, J.; SILVA, M. C. O. Grupo de Orientação a indivíduos acometidos por doenças reumáticas: espaço educativo e terapêutico. Revista Brasileira de Reumatologia, v. 46, n. 2, p. 134-136, 2006. http://dx.doi.org/10.1590/S0482-50042006000200008

DE CARLO, M. M. R. P. et al. Terapia Ocupacional em Reumatologia: princípios e perspectivas. In: DE CARLO, M. M. R. P.; LUZO, M. C. M. Terapia Ocupacional: Reabilitação física e contextos hospitalares. São Paulo: Roca, 2004. p. 153-182.

DE CARLO, M. M. R. P.; ELUI, V. M. C.; PACKER, M. P. Terapia Ocupacional e Atenção a Pacientes com Dor não-oncológica. In: DE CARLO, M. M. R. P.; QUEIROZ, M. E. Dor e cuidados paliativos: Terapia Ocupacional e interdisciplinaridade. São Paulo: Roca, 2007. p. 167-190.

DE CARLO, M. M. R. P.; QUEIROZ, M. E. G.; SANTOS, W. A. Terapia Ocupacional em dor e cuidados paliativos: princípios, modelos de intervençáo e perspectivas. In: DE CARLO, M. M. R. P.; QUEIROZ, M. E. Dor e cuidados paliativos: Terapia Ocupacional e interdisciplinaridade. São Paulo: Roca, 2007. p. 126-145.

ENGEL, J. M. Tratamento para componentes psicossociais: controle da dor. In: NEISTADT, M. E.; CREPEAU, E. B. (Orgs.). Willard, Spackman. Terapia Ocupacional. 9. ed. Rio de Janeiro: Guanabara Koogan, 2002. p. 422-426.

ENGEL, J. M. Controle da dor. In: PEDRETTI, L. W.; EARLY, M. B. Terapia Ocupacional: Capacidades práticas para as disfunções físicas. 5. ed. São Paulo: Roca, 2005. p. 518-525.

FIGUEIRÓ, J. A. A dor. São Paulo: Publifolha, 2000.

GARCIA, J. B. S. Semiologia da dor. Jornal Dor, ano 7, n. 23, p. 3, 2007a. Disponível em: <http:/www.dor. org.br/images/jornal23.pdf>. Acesso em: 15 jun. 2009.

GARCIA, J. B. S. Ano Internacional de combate à dor na Mulher. Jornal Dor, ano 7, n. 26, p. 3, 2007b. Disponível em <http://www.dor.org.br/images/jornal26.pdf>. Acesso em: 15 jun. 2009.

HAGEDORN, R. O processo de Terapia Ocupacional. In: HAGEDORN, R. Fundamentos da prática em Terapia Ocupacional. São Paulo: Dynamis Editorial, 2001. p. 19-27.
McCAHON, S. et al. Self-report and pain behavior among patients with chronic pain. Clinical Journal of Pain, v. 21, n. 3, p. 223-231, 2005. PMid:15818074. http://dx.doi. org/10.1097/00002508-200505000-00005

MELLO, M. A. F. et al. Processo avaliativo em Terapia Ocupacional. In: DE CARLO, M. M. R. P.; LUZO, M. C. M. Terapia Ocupacional: reabilitação física e contextos hospitalares. São Paulo: Roca, 2004. p. 74-98.

MERSKEY, H.; BOGDUK, N. Pain Terminology. The Seattle: International Association for the Study of Pain - IASP, 2007. Disponível em <http://www. iasp-pain.org/AM/Template.cfm?Section=Pain Definitions \&Template $=/ \mathrm{CM} / \mathrm{HTMLDisplay}$. cfm\&ContentID=1728>. Acesso em: 29 ago. 2009.

MIRABELLI-SUSENS, L. Gerenciamento da dor. In: UMPHRED, D. A. Reabilitação Neurológica. 4. ed. Barueri: Manole, 2004. p. 936-961.

MÜLLERSDORF, M.; SÖDERBACK, I. Occupational therapist's assessments of adults with long-term pain: The Swedish experience. Occupational Therapy international, v. 9, n. 1, p. 1-23, 2002. http://dx.doi.org/10.1002/oti.153

NOORDHOEK, J.; LOSCHIAVO, F. Q. Intervenção da Terapia Ocupacional no tratamento de indivíduos com doenças reumáticas utilizando a abordagem da proteção articular. Revista Brasileira de Reumatologia, v. 45, n. 4, p. 242-244, 2005.

NOORDHOEK, J.; JOKL, L. Efeito da música e de exercícios físicos num grupo de pessoas reumáticas: estudo piloto. Acta Fisiatrica, v. 15, n. 2, p. 125-127, 2008.

NUNES, C. M. P.; PENA, L. R. Atuação da Terapia Ocupacional no gerenciamento da dor crônica para o trabalho no complexo do HC/UFMG. In: ENCONTRO DE EXTENSÃO DA UFMG, 8., 2005, Belo Horizonte. Anais... Belo Horizonte: UFMG, 2005.

NUNES, C. M. P. Dor neuromusculoesquelética. In: CAVALCANTI, A.; GALVÃO, C. Terapia Ocupacional: fundamentação e prática. Rio de Janeiro: Guanabara Koogan, 2007. p. 258-268.

PAIVA, E. S. et al. Manejo da dor. Revista Brasileira de Reumatologia, v. 46, n. 4, p. 292-296, 2006.

PEDRETTI, L.; EARLY, M. B. Desempenho ocupacional e modelos de prática para disfunção física. In: PEDRETTI, L. W.; EARLY, M. B. Terapia Ocupacional: capacidades práticas para as disfunçốes físicas. 5. ed. São Paulo: Roca, 2005, p. 3-13

PENGO, M. M. S. B.; SANTOS, W. A. O papel da Terapia Ocupacional em oncologia. In: DE CARLO, M. M. R. P.; LUZO, M. C. M. Terapia Ocupacional: reabilitação física e contextos hospitalares. São Paulo: Roca, 2004. p. 232-255.

RADOMSKI, M. V. Avaliando o contexto: pessoal, social e cultural. In: TROMBLY, C. A.; RADOMSKI, M. V. Terapia Ocupacional para disfunçōes físicas. 5. ed. Sáo Paulo: Santos, 2005. p. 223-224.

SANTANA, J. M. Avaliação do paciente com dor. In: DE CARLO, M. M. R. P.; QUEIROZ, M. E. Dor e cuidados paliativos: Terapia Ocupacional e interdisciplinaridade. São Paulo: Roca, 2007. p. 69-86. 
SPECIALI, J. G.; GONÇALVES, D. A. G. Classificação, Fisiopatologia e Epidemiologia da Dor. In: DE CARLO, M. M. R. P.; QUEIROZ, M. E. Dor e cuidados paliativos: Terapia Ocupacional e interdisciplinaridade. São Paulo: Roca, 2007. p. 27-61.

STANOS, S.; HOULE, T. T. Multidisciplinary and interdisciplinary management of chronic pain. Physical Medicine \& Rehabilitation Clinics of North America, v. 17, n. 2, p. 435-450, 2006. PMid:16616276. http://dx.doi. org/10.1016/j.pmr.2005.12.004

STRONG, J. Assessment of pain perception in clinical practice. Manual Therapy, v. 4, n. 4, p. 216-1220, 1999. PMid:10593110. http://dx.doi.org/10.1054/math.1999.0205
TEIXEIRA, M. J. et al. Epidemiologia da dor. In: TEIXEIRA, M. J.; FIGUEIRÓ, J. A. B. Dor: epidemiologia e evolução histórica da dor. São Paulo: Moreira Junior, 2001. p. 3-9.

TORQUETTI, A. et al. Programas de proteção articular para indivíduos com artrite reumatóide: uma revisão de literatura. Revista de Terapia Ocupacional da Universidade de São Paulo, v. 19, n. 2, p. 76-84, 2008.

TSUKIMOTO, G. R. et al. Avaliação longitudinal da escola de postura para dor lombar crônica através da aplicação dos questionários Roland Morris e Short Form Health Survey (SF-36). Acta Fisiátrica, v. 13, n. 2, p. 63-39, 2006.

\section{Contribuição dos Autores}

Marilles Fuchs: pesquisa bibliográfica nos bancos de dados, concepção do texto manuscrito, seleção e análise dos artigos, redação e revisão do texto. Marina Redekop Cassapian: orientação e revisão do texto.

\section{Notas}

${ }^{1}$ Artigo de pesquisa bibliográfica elaborado a partir da Monografia de Conclusão do Curso de Terapia Ocupacional da UFPR, 2010.

${ }^{2}$ BEECHER, H. Measurement of subjective responses. New York: Oxford University Press, 1959.

${ }^{3}$ YOSIPOVITCH, G.; HUNDLEY, J. L. Practical guidelines for relief of itch. Dermatolology Nursing, v. 16, n. 4, p. 325-328, 2004 .

${ }^{4}$ NORDENSKIÖLD, U.; AlTHOFF, B.; HANSEN, A. Joint Protection - for Activeling GUIDE. Stockholm: The Swedish Rheumatism Association, 1994.

5 STRONG, J. Occupational therapy's contribution to pain management in Queensland. Australian Occupational Therapy Journal, v. 33, p.101-7.

${ }^{6}$ PALMER, P.; SIMONS, J. Joint protection: a critical review. British Journal of Occupational Therapy, v. 54, n. 12, p. 453-458, 1991.

${ }^{7}$ HAMMOND, A. Joint protection behavior in patients with rheumatoid arthritis following an education program. Arthritis Care and Research, v. 7, n. 1, 1994. 